\title{
Effect of triamcinolone added to scalp nerve block for postoperative pain management of Moyamoya disease
}

\author{
Hee-Soo Kim, Seung-Jun Lee, Chong Sung Kim, and Jin-Tae Kim \\ Department of Anesthesiology and Pain Medicine, Seoul National University College of Medicine, Seoul, Korea
}

Ropivacaine is popular for nerve block because of its long duration of action. However, it cannot be used with epinephrine, as it also has a similar vasoconstricive effect. Corticosteroids improve the duration and quality of nerve blockade via an unknown mechanism [1].

Triamcinolone is a popular corticosteroid of high molecular weight and complex structure used for prolonging the duration of action of local anesthetics, but little is known about its effectiveness. We evaluated its addition to local anesthetics with regard to duration of action and morbidity.

After obtaining approval from the institutional review board, written consent from parents was obtained. Subjects were scheduled to undergo encephaloduroarteriosynangiosis (EDAS) for Moyamoya disease between September 18, 2006 and December 31, 2008. All subjects were children aged 2-15 years old, classified ASA $1-2$. Subjects were randomly allocated to one of two groups (Group $\mathrm{R}=20$, Group $\mathrm{T}=19$ ). After unilateral EDAS was done under general anesthesia, we performed scalp nerve blocks, using $0.25 \%$ ropivacaine with epinephrine ( $1: 200,000)$ to rule out vascular injection. In Group T, $0.2 \%$ triamcinolone was added to the ropivacaine. Scalp nerve block was performed at the supraorbital, supratrochlear and auriculotemporal nerves as described by Pinosky et al. [2]. For each nerve block, $1.5 \mathrm{ml}$ of solution was injected. At the end of the procedure, the patient was sent awake to the post anesthesia care unit (PACU) or to the intensive care unit (ICU) after midazolam had been given. We evaluated patients' response for scalp nerve blocks after they had recovered sufficiently to obey commands in the PACU or ICU. When the nerve block failed, we gave routine medication for pain. The time when the patient first complained of pain was recorded. The anesthesiologist visited the children and parents at 8 and 16 hours after the operation to evaluate visual analogue scale (VAS $0-10,>8$ years) or faces pain scale (FPS, $<8$ years). We also reviewed medical records for routine analgesics used during the hospital stay, and incidence of nausea or vomiting. Brain MRI was performed if any signs or symptoms of brain infarction were observed. Patients who recovered from the operation without complications made follow-up visits after 6 months, at which brain MRI scans were performed. Sample size was determined according to a pilot study of 13 patients performed in 2006. The time at which patients first complained of pain was $12.9 \pm 5.3$ hours in group $\mathrm{T}$ and $6.3 \pm 5.2$ hours in group R. If we hypothesize that a difference in block times between groups exists, we would need at least 9 subjects in each group at a significance level of $5 \%$ and power of $80 \%$. We used SPSS 12.0 for statistics analysis; the unpaired T-test was used to compare continuous variables between two groups. Pearson's Chi square was used to determine difference of frequency of nausea, vomiting or brain infarction after the operation. $\mathrm{P}<0.05$ was considered statistically significant.

There was no difference in demographic characteristics, preoperative infarction history, operation time, anesthesia time, PACU stay time, or medication during the postoperative period. Group R patients first complained of pain at 9.2 \pm 9.4 hours and Group $\mathrm{T}$ patients at $14.2 \pm 10.0$ hours $(\mathrm{P}=0.11)$. VAS at 8 hours after surgery was $1.2 \pm 1.5$ in group $\mathrm{R}$ and $1.5 \pm 2.4$ in group $\mathrm{T}$ $(\mathrm{P}=0.56)$; at 16 hours it was $1.2 \pm 1.7$ in group $\mathrm{R}$ and $2.3 \pm 2.8 \mathrm{in}$

Corresponding author: Hee-Soo Kim, M.D., Ph.D., Department of Anesthesiology and Pain Medicine, Seoul National University College of Medicine, 28, Yeongeon-dong, Jongno-gu, Seoul 110-744, Korea. Tel: 82-2-2072-3087, Fax: 82-2-766-3087, E-mail: dami0605@snu.ac.kr (c) This is an open-access article distributed under the terms of the Creative Commons Attribution Non-Commercial License (http:// creativecommons.org/licenses/by-nc/3.0/), which permits unrestricted non-commercial use, distribution, and reproduction in any medium, provided the original work is properly cited. 
group $\mathrm{T}(\mathrm{P}=0.15)$. The incidences of nausea and/or vomiting were 16 in group $\mathrm{R}$ and 10 in group $\mathrm{T}(\mathrm{P}=0.07)$. One patient in each group suffered a new infarct according to MRI within 6 months.

In our study, triamcinolone added to ropivacaine failed to improve the quality and duration of scalp nerve block. Dexamethasone, 4-6 times more potent than triamcinolone, improved the onset or duration of brachial plexus nerve block when added to local anesthetics [1,3]. Ashkenazi reported that triamcinolone failed to improve the duration of greater occipital nerve block with lidocaine and bupivacaine for migraine [4]. Although the dosage of triamcinolone and method of pain evaluation were different from our study, the result is similar, suggesting that triamcinolone is not efficacious. Two complications were considered to be caused by nerve block; one patient could not open his eye due to eyelid swelling associated with the block; the other patient complained of 'dazzling' on the side of the block. Both complications subsided by the following day without sequelae.

\section{References}

1. Movafegh A, Razazian M, Hajimaohamadi F, Meysamie A. Dexamethasone added to lidocaine prolongs axillary brachial plexus blockade. Anesth Analg 2006; 102: 263-7.

2. Pinosky ML, Fishman RL, Reeves ST, Harvey SC, Patel S, Palesch Y, et al. The effect of bupivacaine skull block on the hemodynamic response to craniotomy. Anesth Analg 1996; 83: 1256-61.

3. Shrestha BR, Maharjan SK, Tabedar S. Supraclavicular brachial plexus block with and without dexamethasone - a comparative study. Kathmandu Univ Med J (KUMJ) 2003; 1: 158-60.

4. Ashkenazi A, Matro R, Shaw JW, Abbas MA, Silberstein SD. Greater occipital nerve block using local anaesthetics alone or with triamcinolone for transformed migraine: a randomised comparative study. J Neurol Neurosurg Psychiatry 2008; 79: 415-7. 